\title{
Directed forgetting in monkeys
}

\author{
WILLIAM A. ROBERTS, DWIGHT S. MAZMANIAN, and PHILIPP J. KRAEMER \\ University of Western Ontario, London, Ontario, Canada
}

\begin{abstract}
Based on several recent demonstrations of a directed forgetting effect in pigeons, three experiments were carried out in an attempt to demonstrate directed forgetting in three squirrel monkeys. During initial training with a delayed matching-to-sample procedure, retention tests were always given for sample stimuli followed by remember cues (R-cues) and were always omitted for sample stimuli followed by forget cues (F-cues). Retention of F-cued items was tested on probe trials after initial training. The first two experiments examined the effects of R- and Fcues on memory for slide-projected pictures, with different pictures used on each trial of a session. In Experiment 1, a complex design was used in which one or two sample pictures were presented on each trial; when two pictures were presented, both could be R-cued or F-cued, or one could be R-cued and the other F-cued. A simpler design was used in Experiment 2, with only single pictures presented as sample stimuli and half the trials within a session $R$-cued and the other half F-cued. In both of these experiments, no differential retention of R- and F-cued stimuli was found, even at a retention interval as long as $16 \mathrm{sec}$. In Experiment 3, a series of studies was performed to test for directed forgetting when only two sample stimuli were used repeatedly throughout training and testing. With two pictures as sample stimuli, clear evidence of directed forgetting was found in Experiment $3 \mathrm{~b}$. It is suggested that the directed forgetting effect may arise only when a small set of sample stimuli is used.
\end{abstract}

The phenomenon of directed forgetting was first revealed in human subjects in two experimental paradigms. In one paradigm, memory for items to be forgotten was assessed directly, and in the other paradigm, the effect of a forget instruction was measured indirectly by its effect on intralist interference. In the first type of paradigm, subjects were presented with a list of words on which each word was followed by a remember cue (R-cue) or a forget cue (F-cue). While only memory of the R-cued items was tested initially, the subjects eventually were surprised by a request for recall of both R- and F-cued items. Typically, recall of F-cued words was substantially lower than that of R-cued words (Woodward \& Bjork, 1971). In the second paradigm, the subjects were presented with an initial set of verbal items, were told to forget those items, and then were presented with a final set of items. The forget instruction substantially reduced the proactive interference (PI) that initial items otherwise exerted on the recall of final items (Bjork, 1970; Bjork, LaBerge, \& LeGrande, 1968).

Recently, analogous paradigms for the study of directed forgetting in animals have been developed. The direct measurement of memory for F-cued stimuli has been studied in several experiments with pigeons. In these experiments, birds initially were trained to perform delayed matching-to-sample, with

Support for this research was provided by Grant A7894 from the Natural Sciences and Engineering Research Council of Canada. Address requests for reprints to W. A. Roberts, Department of Psychology, The University of Western Ontario, London, Ontario, Canada N6A SC2. sample stimuli sometimes followed by $R$-cues and sometimes followed by F-cues. For example, red and green sample stimuli presented on a key might be followed by a brief presentation, on the same key, of a vertical white line as an $\mathbf{R}$-cue or of a horizontal line as an F-cue. At the end of the delay or retention interval, a retention test would occur if the sample had been followed by an R-cue but would be omitted if the sample had been followed by an F-cue. Eventually, occasional probe tests would be carried out in which memory for an F-cued sample stimulus would be tested.

Results from this type of experiment have shown consistently that pigeons perform at a higher level of accuracy on R-cued trials than on F-cued probe trials. This effect has been demonstrated with a variety of procedures, which include successive delayed matching (Grant, 1981a) and simultaneous delayed matching using both identical sample and comparison stimuli (Stonebraker, Rilling, \& Kendrick, 1981) and samples of food or no food mapped symbolically onto colored comparison keys (Maki \& Hegvik, 1980; Maki, Olson, \& Rego, 1981). Signals used as Rcues and F-cues have been lines or patterns projected on keys (Kendrick, Rilling, \& Stonebraker, 1981; Stonebraker \& Rilling, 1981), the presence or absence of houselight (Maki \& Hegvik, 1980), and the presentation of differently colored overhead lights (Maki et al., 1981). Furthermore, there is now evidence that the same phenomenon appears in rats when they must remember which alley of a T-maze was entered and different click rates provided $R$ - and F-cues (Grant, 1982). 
Grant recently has carried out experiments with animals analogous to the intralist cuing procedure used with human subjects. In experiments with rats, Grant (1982) forced subjects to enter the two arms of a $T$-maze in succession and then tested retention of memory for the most recently entered arm. This procedure normally generates a strong intratrial PI effect, in that memory for the last-entered arm is much lower than it is on control trials when only a single arm is entered. Grant found that this PI effect was eliminated if an F-cue was introduced after the animal had run down the first arm but before it ran down the second arm. By introducing an F-cue between interfering and target sample stimuli, Grant (in press) found similar alleviation of intratrial PI in delayed matching-to-sample with pigeons.

It has been suggested that $R$ - and F-cues affect retention in animal experiments by controlling the degree of rehearsal of a representation upon which response to the comparison stimuli is based (Grant, 1981a). Rehearsal then is terminated or dampened upon presentation of an F-cue, because memory based on the sample is no longer useful. Some questions about this interpretation have arisen from experiments in which events other than the omission of a retention test have been presented at the end of the delay on F-cued trials. Grant (1981a) reinforced pigeons on $50 \%$ of F-cued trials for pecking a dot placed on the test stimulus at the end of the delay; with this procedure, pigeons showed lower accuracy on F-cued probe trials than on R-cued trials, just as in the omission procedure. However, other investigators have found that the differential accuracy between R-cued trials and F-cued probe trials was attenuated or eliminated by terminating F-cued training trials with a discrimination between irrelevant stimuli (Kendrick et al., 1981; Maki \& Hegvik, 1980), reinforced pecking to an irrelevant pattern (Maki et al., 1981), and response-independent reinforcement (Kendrick et al., 1981). Kendrick et al. have noted that pigeons pecked the sample key throughout the retention interval on both $R$ - and Fcued trials when reinforcement occurred at the end of both types of trials; when F-cues were followed by no reinforcement, pecking occurred only on R-cued trials. They suggest that pecking may establish a context necessary for retrieval of memory of the sample stimulus. The directed forgetting effect then would arise from the absence of a retrieval context when Fcued trials involve the omission of reinforcement. Grant (in press) points out that such an account does not explain the effects of an F-cue on intratrial PI, since both $F$-cued and $R$-cued interfering stimuli are followed by a target sample stimulus and a retention test. However, differential rehearsal of $R$ - and Fcued items would explain the effects of F-cues on intratrial interference.
In this article, we report some experiments carried out to examine the possibility that directed forgetting can be found in monkeys. Since the phenomenon has been found in pigeons and rats, and one implication of these findings is that these species are capable of controlled processing (Maki, 1981), it was anticipated that memory in monkeys also could be controlled by $R$ - and F-cues. The procedures used were generally similar to those that have been used with pigeons. Different visual stimuli acted as R- and Fcues and followed sample stimuli; throughout training, R-cued items were always tested with a delayed matching procedure and F-cued items were never tested. A small proportion of probe trials, on which F-cued sample stimuli were followed by retention tests, eventually were introduced. One departure from the pigeon work was the use of a wide variety of sample and comparison stimuli in Experiments 1 and 2 . The same pair of stimuli were used throughout repeated tests with pigeons, whereas pictorial stimuli drawn from a large pool were used with monkeys and different items appeared on each trial of a session.

\section{EXPERIMENT 1}

A number of experiments on directed forgetting carried out with humans have used an intraserial cuing procedure. That is, within a list of verbal items, some members are followed by R-cues and some are followed by F-cues, and retention may be measured for both kinds of items. One purpose of this experiment was to use an intraserial cuing procedure with monkeys. Since it has been demonstrated that monkeys can retain lists of stimuli (Roberts \& Kraemer, 1981; Sands \& Wright, 1980a, 1980b, 1982), it seemed feasible to use an intraserial cuing procedure with these animals. Lists containing two pictures were presented with both items R-cued, both items F-cued, or one item R-cued and the other item Fcued. When both list members had been R-cued, retention tests for both items followed; with a mixed list, only the R-cued item was tested; and following a list on which both items were F-cued, no retention tests were given. In addition to these two-item lists, sessions contained trials with single items presented and followed either by an $\mathbf{R}$-cue and a retention test or by an F-cue and omission of the retention test. One other variable incorporated into the experiment was retention interval; following lists of one or two sample items and R-and F-cues, the retention test was delayed for 1 or $5 \mathrm{sec}$.

Several effects were looked for in this experiment. A primary concern was whether the directed forgetting phenomenon seen in pigeons and rats would appear. This would be revealed by a significant drop in performance on single-item F-cued probe trials rela- 
tive to R-cued trials. Within the intraserial cuing conditions used here, we were interested in seeing whether directed forgetting within a list could be selective. Given a mixed list, would monkeys forget the F-cued item and remember the $R$-cued item? Another possible effect was that F-cuing one member of a mixed list might alleviate the memory load created by a two-item list (Bjork, 1970) and enhance retention of the $\mathbf{R}$-cued member relative to items contained in a two-item list where both members were $\mathbf{R}$ cued. Finally, it was anticipated that if a directed forgetting effect was found, it might interact with the length of the delay interval, since directed forgetting in pigeons has been found to become more pronounced the longer the interval that elapses after presentation of the F-cue (Maki \& Hegvik, 1980; Maki et al., 1981).

\section{Method}

Subjects. Three adult squirrel monkeys (Saimiri sciureus) were tested. Two monkeys were females (Chick and Dozy), and one was a male (Stumpy). All three monkeys had been trained extensively to perform delayed matching-to-sample with visual stimuli, both black-and-white geometric forms (Roberts \& Kraemer, 1981) and colored slide-projected pictures.

Apparatus. Testing was carried out in a chamber that measured $40.5 \times 29.5 \mathrm{~cm}$ (floor dimensions) $\times 25 \mathrm{~cm}$ (wall height). The sides and top of the chamber were made of transparent acrylic, and the front and back walls were made of sheet metal. The front wall consisted of three 12.5-cm-wide panels, with the side panels bent inward at a 135-deg angle to the center panel. Each of the three panels contained $10 \times 7 \mathrm{~cm}$ transparent acrylic screens, with the bottom edge of each screen $14.5 \mathrm{~cm}$ above the floor of the chamber. Mounted behind each screen was a Kodak Carousel slide projector (Model 650H) with its lens approximately $30 \mathrm{~cm}$ from the screen. Slides projected from the projectors completely filled the acrylic screens. Within the chamber, transparent pecking keys were mounted in front of each screen. The monkeys were transported to the testing chamber in a cage that fit snugly into the chamber and had transparent acrylic panels in front of each screen. A $2.9-\mathrm{cm}$ diam hole cut in each panel allowed the monkeys to press the keys in front of each screen. A magazine that dispensed 190-mg Noyes banana and sucrose pellets was located at the bottom of the center panel. A .1-A 28-V houselight was mounted on the center panel, $2 \mathrm{~cm}$ above the upper edge of the viewing screen.

The sequences of sample and comparison stimuli used in a given session were programmed by sequences of slides placed in 140-slide trays. The slides used were drawn randomly from a pool of 150 slides. The pool contained a wide variety of colored pictures, which fell into categories such as fruits, vegetables, plants, flowers, animals, people, and buildings. Some slides were pictures taken by us of actual objects, and some slides were made by photographing pictures in magazines.

The animals were tested in a darkened room, with control equipment for the apparatus placed in an adjacent room. Response requirements, exposure durations, delays, and intertrial intervals were controlled by relays, counters, and timers that were activated by a paper-tape reader. Printing counters recorded choices on each trial.

Procedure. Each monkey was given 36 sessions of initial testing, with each session containing 32 trials. Within a session, there were four each of R trials, F trials, RF trials, and FR trials, and eight each of FF and RR trials. On R trials, a single sample item was followed by an R-cue and a test for memory of that item. On $F$ trials, a single sample item was followed by an F-cue and termination of the trial without a test. The same procedure was used on FF trials, except that each of two sample stimuli was followed by an F-cue before trial termination. Mixed trials, RF and FR, involved two sample stimuli, with one followed by an R-cue and the other by an F-cue; only the R-cued item was tested after the retention interval. In the case of RR trials, both sample items were tested, with the first item presented tested first, $\mathbf{R}_{\mathbf{1}} \mathbf{R}_{\mathbf{2}}$, on four trials and the second item presented tested first, $\mathbf{R}_{2} \mathbf{R}_{1}$, on four trials. Different trial types occurred in random orders within sessions, and tests of each R-cued position contained the matching slide equally often on the left and right screens.

The 36 sessions of initial testing were broken into three blocks of 12 sessions each. Within a block of sessions, two sets of slide trays were used on alternate days. Thus, monkeys were tested on the same trays on odd and even days (sessions). At the end of a block of sessions, the orders of trial types were rerandomized for both sets of trays, with a new random selection of slides from the pool for use on each trial. On a fourth block of 12 test sessions, each session contained 32 regular trials like those used in initial testing and five probe trials that were randomly intermixed with the regular trials. There was one each of $F, F R$, and RF probe trials and two FF probe trials. On probe trials, the F-cued items were tested. In the case of FF trials, the first item was tested on one trial and the second item was tested on the other trial. On FR and RF probe trials, the F-cued item was always tested first and the R-cued item was tested second. Since two sets of trays were used on alternate days, the left-right positions of the correct comparison stimulus were reversed from one set of trays to the other for each type of probe trial. During both initial training and probe testing, delays of 1 and $5 \mathrm{sec}$ were varied between sessions in a doublealternation pattern, so that each delay was used equally often with each set of slide trays.

Each trial was initiated by a white light projected on the center screen (a transparent slide). Ten presses on the center key were required to advance to the first picture, and another 10 presses had to be completed to advance to the next slide. The slide that followed the picture contained either an $\mathbf{R}$ - or an F-cue and was exposed for 2 sec. The $R$ - and $F$-cues were pictures of the letters $R$ and $F$, which had been photographed as yellow outline figures against a black background. If a single sample stimulus had been presented and followed by an F-cue, the trial terminated at the end of presentation of the F-cue and a 20 -sec intertrial interval spent in darkness began. When a single sample stimulus was followed by an $R$-cue, a 1 - or 5-sec delay spent in darkness followed. The delay terminated with presentation of a matching picture on one side screen and a nonmatching picture on the other side screen. Two presses on the matching side key produced $2 \mathrm{sec}$ of illumination of the houselight, the brief sound of a $2900-\mathrm{Hz}$ tone, and a reward pellet; two presses on the nonmatching side key ended the trial without reward. On trials that involved the presentation of two sample items, each sample was presented until 10 presses were completed on the center key and R-and F-cues followed each item for $2 \mathrm{sec}$. The trial was terminated immediately after the second F-cue on FF trials. On mixed trials, only matching and nonmatching slides for the R-cued item were presented on the side screens after 1- or 5-sec delays. When both sample items were R-cued, the delay ended with presentation of matching and nonmatching pictures for the first item to be tested; following either two presses on the nonmatching item or choice of the matching picture and delivery of reward, a 1sec darkened time-out period was introduced and followed by presentation of matching and nonmatching pictures for the second sample stimulus to be tested. The intertrial interval then followed an incorrect choice or a correct choice and delivery of reward.

\section{Results and Discussion}

Initial training. The percentages of correct matching responses on each type of trial and item tested are presented in Table 1 . The mean percentage of correct responses at each retention interval was 77.5 at $1 \mathrm{sec}$ and 77.6 at $5 \mathrm{sec}$. Since the retention interval 
Table 1

Percentages of Correct Matching Responses Made on Different Types of Trials During Initial Training (Experiment 1)

\begin{tabular}{ccccc}
\hline & \multicolumn{3}{c}{ Subject } & \\
\cline { 2 - 4 } Type of Trial & Stumpy & Chick & Dozy & Mean \\
\hline $\mathbf{R}$ & 79.9 & 88.2 & 87.5 & 85.2 \\
$\mathbf{R}_{1} \mathbf{R}_{2}$ & 84.0 & 78.5 & 76.4 & 79.6 \\
$\overline{\mathbf{R}}_{\mathbf{1}} \mathbf{R}_{2}$ & 72.9 & 63.2 & 70.8 & 69.0 \\
$\mathbf{R}_{2} \overline{\mathbf{R}}_{1}$ & 61.1 & 64.6 & 72.2 & 66.0 \\
$\mathbf{R}_{2} \mathbf{R}_{1}$ & 88.9 & 83.3 & 86.1 & 86.1 \\
$\mathbf{F R}_{\mathbf{R}}$ & 81.9 & 70.8 & 88.2 & 80.3 \\
$\mathbf{R F}$ & 77.1 & 77.8 & 75.7 & 76.8 \\
\hline
\end{tabular}

Note-For $R_{1} R_{2}$ and $R_{2} R_{1}$ trials, the score reported is for the underlined item.

had no effect on performance $(F<1.0)$ and did not interact with types of trials, the data were averaged over this factor. Examination of both the scores for individual subjects and the mean scores suggests that retention was strongly affected by output interference and less strongly by input interference. These effects can be found by examining performance on RR trials; the scores in Table 1 on different types of $R R$ trials show accuracy for the underlined member of the pair. Within RR trials, the item tested first was considerably better retained than the item tested second, with input position equated. Therefore, $\underline{R}_{1} \mathbf{R}_{2}$ was superior to $\mathbf{R}_{2} \mathbf{R}_{1}$, and $\mathbf{R}_{2} \mathbf{R}_{1}$ was superior to $\mathbf{R}_{\mathbf{1}} \mathbf{R}_{2}$. Evidence for a recency effect comes from the observation that the second item presented was retained better than the first item presented, with output position equated; that is, $\mathbf{R}_{\mathbf{2}} \mathbf{R}_{\mathbf{1}}$ was superior to $\underline{R}_{1} \mathbf{R}_{\mathbf{2}}$, and $\mathbf{R}_{\mathbf{1}} \underline{R}_{\mathbf{2}}$ was superior to $\underline{R}_{\mathbf{2}} \bar{R}_{\mathbf{1}}$. There is no suggestion in the data that presenting two items caused forgetting by increasing the memory load, since mean performance was slightly better on $\mathbf{R}_{\mathbf{2}} \mathbf{R}_{\mathbf{1}}$ tests than on $\mathbf{R}$ trials. Finally, the presence of an $F$-cued item did not appear to aid the retention of R-cued items on mixed trials; animals performed somewhat better on $\mathbf{R}_{\mathbf{2}} \mathbf{R}_{\mathbf{1}}$ tests than on FR trials and on $\underline{R}_{1} \mathbf{R}_{\mathbf{2}}$ tests than on $\mathbf{R F}$ trials.

An analysis of variance across the seven types of trials and tests within trials shown in Table 1 yielded a significant effect $[F(6,12)=7.15, p<.05]$. Comparisons between scores using the Newman-Keuls test $(p=.05)$ indicated that $R_{2} R_{1}$, was significantly lower than all other scores, except $\mathbf{R}_{\mathbf{1}} \mathbf{R}_{2}$, and that $\mathbf{R}_{\mathbf{1}} \mathbf{R}_{\mathbf{2}}$ was significantly lower than $R$ and $R_{2} R_{1}$. These results support the importance of output interference when two items are tested on the same trial.

Probe testing. Performance on regular R-cued trials, like those used in initial training, is shown in the top part of Table 2, and performance on F-cued probe trials is shown in the bottom section of the table. On R-cued trials, the mean percentages of correct responses at 1 - and 5-sec retention intervals were 82.9 and 81.8, respectively. Retention interval was not a significant factor ( $F<1.0$ ), and the data presented were averaged over this variable. The mean scores for types of $\mathbf{R}$-cued trials and items within trials show a pattern similar to that seen in Table 1 . For example, performance was clearly worst for the $R_{2} R_{1}$ item, the case in which the first item of a twoitem list was tested last. The effect of types of trials and items tested fell short of statistical significance $[F(6,12)=3.85, p>.05]$

On F-cued probe trials, the mean percentages of correct responses were 87.8 at the 1 -sec delay and 83.3 at the 5-sec delay. These means did not differ significantly $[F(1,2)=4.00, p>.05]$, and the data shown for F-cued trials also have been averaged over the two retention intervals. The means for the F-cued probe trials do not appear to differ greatly among themselves, and this impression was confirmed statistically $(F<1.0)$.

In a final analysis, performance on R-cued and Fcued trials was compared. The data in Table 2 suggest that subjects did only slightly worse on F-cued trials than on R-cued trials. The F-cued trials were divided into three categories, F trials, FF and FR trials, and FF and RF trials. The second category contains trials in which the first of two items presented was F-cued and was tested immediately, and the third category contains trials in which the second of two items presented was F-cued and was tested immediately. The corresponding categories of $\mathbf{R}$-cued trials were $R, R_{1} R_{2}$, and $R_{2} \underline{R}_{1}$. A categories $\times$ cue $X$ subjects analysis of variance yielded no significant effects of categories $[F(2,4)=2.09, p>.05], R$-cue vs. F-cue $(F<1.0)$, or the categories $\times$ cue interaction $(\mathrm{F}<1.0)$.

These findings provide no basis for concluding that $\mathrm{F}$-cues promote loss of memory in monkeys. In

Table 2

Percen tages of Correct Matching Responses Made On Regular R-Cued and on F-Cued Trials (Experiment 1)

\begin{tabular}{|c|c|c|c|c|}
\hline \multirow[b]{2}{*}{ Type of Trial } & \multicolumn{3}{|c|}{ Subject } & \multirow[b]{2}{*}{ Mean } \\
\hline & Stumpy & Chick & Dozy & \\
\hline \multicolumn{5}{|c|}{ Regular R-Cued Trials } \\
\hline $\begin{array}{l}\mathrm{R} \\
\mathrm{R}_{1} \mathbf{R}_{2} \\
\overline{\mathrm{R}}_{1}{ }_{2} \\
\mathrm{R}_{2} \\
\bar{R}_{2} \overline{\mathrm{R}}_{1} \\
\mathrm{R}_{2} \underline{\mathrm{R}}_{1} \\
\mathrm{FR} \\
\mathrm{RF}\end{array}$ & $\begin{array}{l}83.4 \\
85.4 \\
93.8 \\
56.2 \\
93.8 \\
83.4 \\
73.0\end{array}$ & $\begin{array}{l}97.9 \\
95.8 \\
66.7 \\
56.2 \\
72.9 \\
81.2 \\
89.6\end{array}$ & $\begin{array}{l}95.8 \\
89.6 \\
79.2 \\
70.8 \\
91.7 \\
91.6 \\
87.5\end{array}$ & $\begin{array}{l}92.4 \\
90.3 \\
80.0 \\
61.1 \\
86.1 \\
85.4 \\
83.4\end{array}$ \\
\hline \multicolumn{5}{|c|}{ F-Cued Probe Trials } \\
\hline $\begin{array}{l}\text { F } \\
\text { FF } \\
\overline{F F} \\
F \bar{R} \\
R F\end{array}$ & $\begin{array}{r}100.0 \\
75.0 \\
91.6 \\
83.3 \\
66.6\end{array}$ & $\begin{array}{r}91.6 \\
91.6 \\
75.0 \\
91.6 \\
100.0\end{array}$ & $\begin{array}{l}83.4 \\
83.3 \\
83.3 \\
91.6 \\
75.0\end{array}$ & $\begin{array}{l}91.7 \\
83.3 \\
83.3 \\
88.8 \\
80.5\end{array}$ \\
\hline
\end{tabular}

Note-For $R_{1} R_{2}, R_{2} R_{1}$, and FF trials, the score reported is for the underlined item. 
contrast to the robust difference in performance between R-cued trials and F-cued probe trials seen in pigeons, monkeys' performance was not differentially affected by $R$ - and F-cues.

\section{EXPERIMENT 2}

In retrospect, it was seen that certain problems with Experiment 1 may have prevented a demonstration of directed forgetting. One possible problem may have been that the experiment was too complex. In addition to the use of single item R- and F-cued trials, the use of trials containing two pictures, with both R-cued or F-cued or with one R-cued and the other F-cued, may have confused subjects and caused lack of attention to R- and F-cues. A second potential problem may have been the choice of $R$ - and $F$ cues. The letters $R$ and $F$ have somewhat similar configurations, and it is possible that they were not discriminated as different stimuli by the monkeys. Some evidence of the discriminability of R-and F-cues, independent of matching accuracy, would be desirable. Finally, it is possible that a directed forgetting effect would be seen over a longer retention interval than was used in Experiment 1. Perhaps F-cues did direct the forgetting of the preceding picture, but retention intervals of 1 to $5 \mathrm{sec}$ were not long enough to allow substantial forgetting to take place.

Experiment 2 was carried out as a further attempt to demonstrate directed forgetting in monkeys, and the procedure was modified to deal with the various problems which may have been present in Experiment 1 . Only trials containing single sample stimuli were used, with half the items R-cued and half the items F-cued during initial training. This procedure then is the same as that which has been used with pigeons and avoids any possible confusion that might arise from the additional use of two-item trials with mixed and unmixed R- and F-cues. Another modification was the use of presumably more discriminable $R$ - and F-cues. The letter F used in Experiment 1 was retained as an $F$-cue, but a homogeneous colored field was used as the R-cue. As a possible measure for detecting the discrimination of R- and F-cues, number of presses on the center screen was recorded during the presentation of $R$ - and F-cues. The possibility that directed forgetting might appear at a longer retention interval than was used in the first experiment was assessed by using retention intervals of 1,8 , and $16 \mathrm{sec}$.

\section{Method}

Subjects. The same three monkeys used in Experiment 1 were used in Experiment 2.

Apparatus. The same equipment and pool of 150 slides used in the first experiment were used again. The F-cue was the yellow letter $F$ on a black background, and the R-cue was a homogeneous light green field.

Procedure. Initial training consisted of 24 training sessions, with each session containing $\mathbf{4 0}$ trials. Within a daily session, 20 trials involved the presentation of a picture followed by an F-cue and trial termination and 20 trials involved the presentation of a picture followed by an $\mathbf{R}$-cue and a retention test after a retention interval. As in Experiment 1, the R-cues and F-cues immediately followed the sample picture and were exposed for $2 \mathrm{sec}$. Trials containing $R$-cues and F-cues were randomly intermixed, and the matching test item was equally often on the left and right screens on R-cued trials. Two sets of slide trays were used on alternate sessions during the first 12 sessions. The orders of R-and F-cued trials and the assignment of slides to trials then were rerandomized for the next block of 12 sessions. Between sessions, retention intervals of 1,8 , and $16 \mathrm{sec}$ were each used on eight sessions and were used equally often with each set of slide trays. Presses on the center screen during presentation of $\mathbf{R}$ - and $\mathbf{F}$-cues were recorded on cumulative counters.

In a third block of 12 sessions, the animals received daily sessions containing $20 \mathrm{R}$-cued trials and $20 \mathrm{~F}$-cued trials on which the retention test was omitted. On four extra probe trials, randomly interspersed with the other trials, an F-cue followed the sample picture and was followed by a retention test. Two sets of slide trays again were used on alternate days, and the matching picture was equally often on the right and left screens on probe trials. Over the 12 sessions, retention intervals of 1,8 , and $16 \mathrm{sec}$ each were used on four sessions.

\section{Results and Discussion}

Initial training. For the 24 sessions of initial training, the percentages of correct responses on $\mathbf{R}$-cued trials are shown in Table 3 for each subject. There is some decline in performance between the retention intervals of 1 and $16 \mathrm{sec}$, but retention interval did not have a significant effect on performance $[F(2,4)$ $=3.27, \mathrm{p}>.05]$.

The mean number of presses per trial made during the 2-sec exposure of the R-cue was 4.74, 2.54, and 1.96 for Stumpy, Chick, and Dozy, respectively; the mean presses on the F-cue for the same animals were $1.01,2.28$, and .80 , respectively. Although each animal pressed the R-cue more than the F-cue, the effect of cue on mean presses was not significant $[F(1,2)=$ $2.73, p>.05]$. The data for separate animals suggest that Stumpy and Dozy did respond differentially to $R$ - and F-cues, whereas Chick did not. Stumpy responded more frequently to the $R$-cue than to the $F$ -

Table 3

Percentages of Correct Matching Responses Made on R-Cued and F-Cued Trials During Initial Training and Probe Trials (Experiment 2)

\begin{tabular}{|c|c|c|c|c|c|}
\hline \multirow[b]{2}{*}{ Cue } & \multirow{2}{*}{$\begin{array}{c}\text { Retention } \\
\text { Interval }\end{array}$} & \multicolumn{3}{|c|}{ Subject } & \multirow[b]{2}{*}{ Mean } \\
\hline & & Stumpy & Chick & Dozy & \\
\hline \multicolumn{6}{|c|}{ Initial Training } \\
\hline R-Cued & $\begin{array}{r}1 \\
8 \\
16\end{array}$ & $\begin{array}{l}90.6 \\
94.4 \\
79.4\end{array}$ & $\begin{array}{l}88.8 \\
87.5 \\
80.0\end{array}$ & $\begin{array}{l}95.6 \\
80.6 \\
82.5\end{array}$ & $\begin{array}{l}91.7 \\
87.5 \\
80.6\end{array}$ \\
\hline \multicolumn{6}{|c|}{ Probe Testing } \\
\hline R-Cued & $\begin{array}{r}1 \\
8 \\
16\end{array}$ & $\begin{array}{l}95.4 \\
85.2 \\
80.7\end{array}$ & $\begin{array}{l}93.2 \\
84.1 \\
92.0\end{array}$ & $\begin{array}{l}97.8 \\
94.3 \\
85.2\end{array}$ & $\begin{array}{l}95.5 \\
87.9 \\
86.0\end{array}$ \\
\hline F-Cued & $\begin{array}{r}1 \\
8 \\
16\end{array}$ & $\begin{array}{l}87.5 \\
81.2 \\
93.8\end{array}$ & $\begin{array}{r}100.0 \\
93.8 \\
100.0\end{array}$ & $\begin{array}{l}93.8 \\
93.8 \\
93.8\end{array}$ & $\begin{array}{l}93.8 \\
89.6 \\
95.9\end{array}$ \\
\hline
\end{tabular}

Note-Retention interval given in seconds. 
cue in every session $[F(1,23)=1,414.24, p<.01]$, with the lowest mean number of presses on the R-cue within a session being 3.60 and the highest mean number of presses on the F-cue being 1.95. Dozy made more mean responses to the $R$-cue than to the F-cue on $17 / 24$ sessions, and the mean difference in responding to cues was significant $[F(1,23)=21.26$, $\mathrm{p}<.01]$. Chick, on the other hand, pressed the R-cue more than the F-cue on only $13 / 24$ sessions and showed no significant difference in mean responses to cues $[F(1,23)=1.12, p>.05]$.

Probe testing. The percentages of correct responses made over the 12 sessions of probe testing are shown in the bottom section of Table 3. The outstanding finding revealed by these data is that the animals performed no worse on F-cued trials than on R-cued trials. In fact, all three monkeys scored slightly better overall on F-cued trials than on R-cued trials. Performance dropped somewhat as a function of the retention interval on $\mathbf{R}$-cued trials, but mean matching accuracy was actually better at the $16-\mathrm{sec}$ retention interval than at the 1- and 8-sec retention intervals on F-cued trials. A cue $\times$ retention interval $X$ subjects analysis of variance showed that there was no significant effect of cue $[F(1,2)=1.84, p>.05]$, retention interval $[F(2,4)=2.77, p>.05]$, or of the cue $\times$ retention interval interaction $[F(2,4)=3.40$, $\mathrm{p}>$.05].

During probe testing, 4.38, 5.21, and .75 mean presses per trial were recorded on the R-cue for Stumpy, Chick, and Dozy, respectively; the corresponding mean number of presses on the F-cue were $.78,1.77$, and .62 , respectively. The difference between mean presses on R-cues and F-cues was not significant $[F(1,2)=4.46, p>.05]$. However, analysis of the data for individual subjects revealed significant differences between $R$-cues and $F$-cues for Stumpy $[F(1,11)=170.00, p<.01]$ and Chick $[F(1,11)=253.46, p<.01]$. As was the case in initial training, Stumpy responded considerably more frequently on the R-cue than on the F-cue in all 12 sessions. Although Chick had shown only slightly greater responding to the $R$-cue in initial training, she, too, pressed the $R$-cue at a much higher rate than she did the F-cue during all 12 sessions of probe testing. Dozy's overall rate of pressing on R- and F-cues fell to a low level during probe testing, and no significant difference between cues was found $(F<1.00)$.

Although this experiment was made considerably simpler in design than Experiment 1, still no evidence of directed forgetting was found, even when the retention interval extended to $16 \mathrm{sec}$. Highly discriminable R- and F-cues were used, and differential responding on these cues throughout the experiment for Stumpy and during probe testing for Chick indicated that these animals discriminated between $\mathbf{R}$ and F-cues. Yet, these animals showed equal or better performance on F-cued trials than on $R$-cued trials and did not differ in performance on $R$ - and Fcued trials from Dozy, who did not respond at different rates to $R$ - and F-cues during probe testing.

\section{EXPERIMENT 3}

The results of Experiments 1 and 2 suggest a striking difference between pigeons and monkeys in the effects of F-cues on retention. With pigeons, following F-cued sample stimuli with omission of the comparison stimuli leads to a large drop in retention relative to R-cued items, when probe tests are given. Thus far, our experiments suggest that monkeys' retention of sample pictures is not in the least affected by F-cues.

One major difference between the experiments done with pigeons and the monkey experiments thus far reported still exists. Pigeons have been tested using only two sample stimuli, usually colored fields or simple geometric black-and-white patterns. By contrast, monkeys were tested with complex colored pictures and with different samples presented on each trial. It is possible that the use of only two relatively noncomplex stimuli is necessary to produce the directed forgetting effect. In order to test this possiblity, a series of further experiments was carried out. Since these experiments were closely related to one another and followed in a logical sequence, they are referred to as Experiments 3a, 3b, and 3c.

\section{EXPERIMENT 3a}

In this experiment, the monkeys were trained and tested with only two colored geometric patterns serving as sample and comparison stimuli. Only two relatively simple stimuli were used, which should allow a test of directed forgetting in monkeys under conditions very close to those used with pigeons.

\section{Method}

Subjects. The subjects were the same monkeys used in the first two experiments.

Apparatus. The same apparatus used in the first two experiments was used in this experiment. In place of the 150-slide pool used in the earlier experiments, only two stimulus items were used. These stimuli were a yellow circle and a light blue rectangle, both photographed against a black background. When projected on the viewing screens, the circle was $5 \mathrm{~cm}$ in diameter and the rectangle measured $5.2 \times 2.6 \mathrm{~cm}$.

Procedure. Initial training consisted of 20 sessions of delayed matching trials, with 40 trials in each session. Within a session, 20 trials were $\mathbf{R}$-cued and 20 trials were $\mathbf{F}$-cued; the yellow circle and the blue rectangle each were followed by an $R$-cue on 10 trials and by an F-cue on 10 trials. The $R$ - and F-cues were the same stimuli as those used in Experiment 2.

Two different 40-trial sequences of randomly ordered $\mathbf{R}$-cued and F-cued trials were used on alternate sessions. On F-cued trials, the sample stimulus was followed immediately by the F-cue for 2 sec and the comparison stimuli were omitted. On R-cued trials, the R-cue was presented for 2 sec immediately after the sample stimulus and was followed after a 1-sec retention interval by the matching and nonmatching comparison stimuli. On R-cued trials, 
the circle and rectangle each appeared equally often on the left and right screens as the matching comparison stimuli. Because preliminary testing had indicated that matching accuracy was low in this task, the retention interval stayed constant at $1 \mathrm{sec}$ throughout the experiment.

After initial training, 12 sessions of probe testing were given. Each session consisted of $\mathbf{4 0}$ R-cued and F-cued trials, identical to those given in initial training. Four probe trials were inserted throughout the 40 regular trials. On two probe trials, the circle was followed by an F-cue and then by a retention test, with the matching circle on the left screen on one test and on the right screen on the other test; on the other two probe trials, the rectangle was followed by an F-cue and a retention test, with the rectangle on the left screen on one test and on the right screen on the other test. As in Experiment 2, the frequency of presses on the R-cue and the $F$ cue was recorded on every session during both initial training and probe testing.

\section{Results and Discussion}

Initial training. The results from the 20 sessions of initial training are shown in the top row of Table 4. It can be seen that the level of matching accuracy was substantially worse in this experiment than it was in the previous two experiments. This observation agrees with previous findings that monkeys transferred from delayed matching testing with a large pool of items to testing with only a few items show a substantial decrement in accuracy (Overman \& Doty, 1980; Sands \& Wright, 1980a, 1980b).

The mean presses per trial on the R-cue for Stumpy, Chick, and Dozy were 4.46, 5.98, and .18, respectively; the mean presses on the F-cue were $1.16,1.32$, and .54 , respectively. The mean $R$-cue and $F$-cue responses averaged over the three animals did not differ significantly $[F(1,2)=2.86, p<.05]$. However, as was the case during probe testing in Experiment 2 , significant differences in mean responses to $R$ - and $F$-cues were found for Stumpy $[F(1,19)$ $=294.32, p<.01]$ and Chick $[F(1,19)=775.57$, $\mathrm{p}<.01$ ], with both monkeys responding more frequently to the R-cue on all 20 sessions. Dozy responded at a low frequency to both cues and made more responses to the F-cue than to the $R$-cue.

Probe testing. The percentages of correct responses on R-cued trials and F-cued probe trials is shown in the bottom section of Table 4. Once again, there is no suggestion of a directed forgetting effect,

Table 4

Percentages of Correct Matching Responses Made on R-Cued and F-Cued Trials During Initial Training and Probe Trials (Experiment 3a)

\begin{tabular}{ccccr}
\hline & \multicolumn{3}{c}{ Subject } & \\
\cline { 3 - 4 } Cue & Stumpy & Chick & Dozy & Mean \\
\hline \multirow{3}{*}{ R-Cued } & 63.8 & Initial Training & \\
& & 68.5 & 55.8 & 62.7 \\
R-Cued & 62.5 & Probe Testing & & \\
F-Cued & 79.2 & 64.2 & 55.8 & 60.8 \\
\hline
\end{tabular}

with the mean percentage of correct responses actually being higher for F-cued trials than for R-cued trials. The difference between percentage of correct responses on $\mathbf{R}$-cued and F-cued trials was not significant $[F(1,2)=1.46, p>.05]$.

The pattern of responding to $R$ - and F-cues during probe testing was much the same as it was during initial training. Stumpy, Chick, and Dozy made 3.46, 3.09 , and .70 mean presses per trial on the R-cue, respectively, and $1.44,1.06$, and .57 mean presses per trial on the F-cue, respectively. Mean responses averaged across the three animals did not differ significantly between $R$ - and $F$-cues $[F(1,2)=4.85, p<.05]$. Analyses for individual subjects revealed significant differences between mean presses to $R$ - and F-cues for Stumpy $[F(1,11)=59.85, p<.01]$ and Chick $[F(1,11)=91.48, p<.01]$, with both monkeys responding more frequently to the $R$-cue than to the $F$ cue on all 12 sessions. Dozy continued to respond at a low rate to both $R$ - and F-cues and showed no significant difference between them $(F<1.00)$.

When only two sample stimuli were used, matching accuracy dropped considerably below that found in Experiments 1 and 2. Within the limitations established by this low level of performance, the results were in complete agreement with those of the first two experiments in showing no evidence of a directed forgetting effect. As in Experiment 2, two monkeys, Stumpy and Chick, showed a highly reliable tendency to respond differently to R- and F-cues, but they were no less accurate on retention tests following $F$ cues than on tests following R-cues.

\section{EXPERIMENT 3b}

A major problem with interpreting the findings from Experiment $3 a$ is the low level of accuracy achieved on R-cued trials. Since subjects achieved only slightly better than $60 \%$ correct responses on $R$-cued trials, a floor effect may have prohibited the observation of a directed forgetting effect. The low level of accuracy in Experiment 3a could have arisen from PI caused by the use of only two sample stimuli (Sands $\&$ Wright, 1980a, 1980b). Another reason for the decline in accuracy from the first two experiments to Experiment 3a could have been the change in the type of stimuli used. Monkeys might have developed a set to match complex, multicolored pictures in the first two experiments and not have been able to transfer this set well to the relatively simple geometric patterns used in Experiment 3a. In Experiment 3b, a further test of directed forgetting was performed with only two sample and comparison stimuli, but the stimuli were two pictures drawn from the pool of 150 slides. It was anticipated that these stimuli might allow the monkeys to reach a higher level of accuracy on R-cued trials and thus allow for a more adequate test of directed forgetting. 


\section{Method}

The subjects and apparatus used in the first three experiments were used again in Experiment $3 \mathrm{~b}$. The sample and comparison stimuli were two slides drawn from the pool of 150 slides, a picture of a red chair and a picture of a green plant. The subjects were given 30 sessions of initial training. Within each session, there were $20 \mathrm{R}$-cued trials and $20 \mathrm{~F}$-cued trials. The two stimuli were used equally often on $\mathbf{R}$-cued and F-cued trials as the sample stimulus and the incorrect comparison stimulus. Two different sets of slide trays, which contained different random orders of trials, were used on alternate days throughout the $\mathbf{3 0}$ days of training. The procedures in effect on R-cued and F-cued trials were exactly the same as those used in Experiments 2 and 3a. Two delays were used, 1 and $5 \mathrm{sec}$; each delay was used on all trials within a session, and the length of delay varied between sessions according to a doublealternation pattern.

After completion of initial training, monkeys were given 30 more sessions of probe testing. On these test sessions, there were 20 trials each of regular R-cued and F-cued trials and four additional probe trials. On probe trials, an F-cue followed the sample stimulus and then was followed by presentation of comparison stimuli. The plant picture appeared as the sample stimulus on two probe trials within each session, and the chair picture appeared on the other two probe trials. With two monkeys, Chick and Dozy, a 1 -sec delay was used throughout testing; the 5-sec delay was not used with these animals because they had not scored sufficiently well at the longer delay in initial training. Stumpy, on the other hand, had shown a relatively high level of accuracy at the 5-sec delay, and was tested at both 1- and 5-sec delays, in the same manner as in initial training. As in initial training, there were two different random orders of trials that were used on alternate sessions.

\section{Results and Discussion}

Initial training. The percentages of correct responses on R-cued trials in initial training are shown in the top two rows of Table 5 . The use of two pictures from the slide pool was successful in raising accuracy above that found in Experiment $3 a$ at a 1sec delay. However, the monkeys still were not as accurate as they had been with multiple sample stimuli in Experiment 2. At the 5-sec delay, Stumpy's per-

Table 5

Percentages of Correct Matching Responses Made on R-Cued and F-Cued Trials During Initial Training and Probe Trials (Experiment 3b)

\begin{tabular}{cccccc}
\hline & $\begin{array}{c}c \\
\text { Retention } \\
\text { Interval }\end{array}$ & Stumpy & Chick & Dozy & Mean \\
\cline { 3 - 5 } R-Cued & 1 & 83.4 & 78.9 & 74.7 & 79.0 \\
& 5 & 81.7 & 65.7 & 64.3 & 70.6 \\
R-Cued & 1 & 77.1 & 71.7 & 69.7 & 72.8 \\
& 5 & 76.2 & & & \\
F-Cued & 1 & 71.4 & 58.3 & 66.7 & 65.5 \\
& 5 & 68.8 & & & \\
R-Cued & 1 & 88.1 & 74.3 & 71.7 & 78.0 \\
& 5 & 77.8 & & & \\
F-Cued & 1 & 65.6 & 53.3 & 60.0 & 59.6 \\
& 5 & 60.7 & & & \\
\hline
\end{tabular}

Note-Retention interval given in seconds. formance dropped only slightly from the 1-sec delay, but both Chick and Dozy showed more substantial losses of accuracy. A test of the difference between the means at 1 - and 5 -sec delays failed to yield a significant effect $[F(1,2)=5.95, p>.05]$.

Mean presses on the R- and F-cues were recorded. On the first 13 sessions, Stumpy responded as he had during Experiments 2 and 3a; the mean responses to the $\mathrm{R}$ - and F-cues were 2.84 and .78, respectively, and the difference between these means was highly significant $[F(1,12)=30.36, p<.01]$. On the remaining sessions, 14-30, Stumpy's rate of responding to the R-cue became negligible and dropped to a mean of .10 , while his responding to the F-cue continued at a moderately low rate of .84 presses per trial. Stumpy now pressed the F-cue significantly more frequently than the $R$-cue $[F(1,16)=66.91, p<.01]$. No reason for this reversal of responding to cues was apparent; however, responding at a low rate to both $\mathbf{R}$ - and Fcues represents appropriate behavior, since there is no causal connection between responding to these cues and trial outcome. Chick continued to respond more to the R-cue than to the F-cue on every session and made 4.48 mean responses per trial to the $R$ cue and .98 mean responses to the F-cue; these means differed significantly $[F(1,29)=309.76, p<.01]$. Dozy continued to respond at low and nondifferential rates to $R$ - and F-cues; her mean responses per trial were .98 to the R-cue and .80 to the F-cue, and these means did not differ significantly $[F(1,29)=$ $3.10, \mathrm{p}>.05]$.

Probe testing. In Table 5, the percentages of correct responses on $\mathbf{R}$ - and $\mathbf{F}$-cued trials are presented separately for Sessions 1-15 and 16-30. A tendency for monkeys to be less accurate on F-cued trials than on R-cued trials was only midly apparent during the first half of the testing sessions but became strongly apparent during the last half of the sessions. On Sessions 1-15, the mean percentages of correct responses differed by about seven percentage points in favor of the R-cued trials over the F-cued trials, but this difference was not significant $[F(1,2)=5.57, p>.05]$. For individual animals, the difference between accuracy on R-cued and F-cued trials was significant in the case of Chick $[F(1,14)=7.13, p<.05]$, but not in the case of Dozy $(F<1.0)$ or of Stumpy at either the 1 -sec or 5-sec delay $(F<1.0)$.

The data from Sessions 16-30 show clear evidence of a directed forgetting effect in all three monkeys. The mean percentages of correct responses differed significantly between $R$ - and F-cued trials $[F(1,2)=29.70, p<.05]$. Analysis of the accuracy scores for individual monkeys showed significant differences between R- and F-cued trials at the 1-sec delay for Stumpy $[F(1,7)=10.50, p<.05]$, Chick $[F(1,14)=28.84, p<.01]$, and Dozy $[F(1,14)=5.24$, $\mathrm{p}<.05]$. The difference seen between Stumpy's scores on R- and F-cued trials at the 5-sec delay fell short of significance $[F(1,6)=3.50, p>.05]$. 
The mean pressses recorded on $R$ - and F-cues showed much the same pattern as was seen in initial training. Stumpy made .83 mean presses per trial to the F-cue but only .01 mean presses on the R-cue; this difference between responding to $\mathbf{R}$ - and F-cues was highly significant $[F(1,29)=207.07, p<.01]$. Chick continued to respond more frequently to the R-cue than to the F-cue, 3.56 vs. 1.12 mean responses, and this difference was significant $[F(1,29)=169.52, p<$ $.01]$. Dozy now showed a tendency to respond more rapidly on the F-cue, .82 mean responses, than on the $\mathrm{R}$-cue, .58 mean responses, and this difference was significant $[F(1,29)=13.91, p<.01]$.

For the first time in this series of experiments, directed forgetting has been demonstrated. These findings suggest that monkeys, like pigeons, learn to deal differently with memories of sample stimuli followed by R- and F-cues. Furthermore, these experiments, taken as a whole, indicate that evidence of directed forgetting in monkeys may be found only when a small set of sample stimuli is used and a reasonably high level of accuracy on R-cued trials is achieved.

\section{EXPERIMENT 3c}

Since a directed forgetting effect was not found in Experiments 1 and 2, with multiple sample stimuli, but was found in Experiment $3 b$, with only two sample stimuli, it may be inferred that the use of a small set of stimuli is necessary to demonstrate the effect. It might be objected, however, that the directed forgetting effect seen in Experiment $3 b$ appeared not because only two sample stimuli were used, but because monkeys only began to process the memories of sample stimuli followed by R- and Fcues differently at that time. That is, all of the practice monkeys had in Experiments 1, 2, and 3a might have been required for animals to begin to initiate different remembering and forgetting processes in Experiment $3 \mathrm{~b}$. If this is the case, then, we should find a directed forgetting effect when subjects are retested with multiple sample stimuli. This possibility was examined in Experiment 3c by giving the monkeys 10 sessions of probe testing equivalent to those used in Experiment 2.

\section{Method}

The subjects and apparatus were the same as those used in the preceding experiments. Each monkey was tested in 10 sessions, with each session containing 44 trials. Of these 44 trials, 20 trials were $R$-cued trials in which the sample stimulus was followed by an $R$-cue and a retention test; another 20 trials were regular F-cued trials in which the sample was followed by an F-cue and the retention test was omitted. On four probe trials, comparison stimuli followed the sample and an F-cue. Of critical importance, different sample and comparison slides drawn from the 150-slide pool were used on each trial. Two different random orders of types of trials and stimulus items alternated between sessions. As in Experiment $3 b$, Chick and Dozy always were tested at a 1-sec delay;
Stumpy was tested at a 1-sec delay on half the sessions and at a 5sec delay on the other half of the sessions.

\section{Results and Discussion}

Two observations were notable in the data from Experiment 3c: Accuracy increased substantially over that found in Experiments $3 a$ and $3 b$, and the directed forgetting effect disappeared. The mean percentages of correct responses at the 1-sec delay were 91.8 on R-cued trials and 89.2 on F-cued probe trials, and the difference between these means did not approach significance $(F<1.0)$. Stumpy made $93.6 \%$ and $85.0 \%$ correct responses on R-cued and F-cued trials, respectively, at the $1-\mathrm{sec}$ delay and $87.2 \%$ and $85.0 \%$ correct responses on R-cued and F-cued trials at the 5-sec delay; neither of these differences between $R$ - and F-cued trials was significant $(F<$ 1.0). Chick was correct on $88.2 \%$ of the R-cued trials and $92.5 \%$ of the F-cued trials, and this difference was not significant $[F(1,9)=1.21, p>.05]$. Finally, Dozy was correct on $93.6 \%$ of the R-cued trials and $90.0 \%$ of the F-cued trials, and these values did not differ significantly $(F<1.0)$.

During presentation of the R- and F-cues, Stumpy never pressed the $R$-cue and made .85 presses per trial on the F-cue; the difference was highly significant $[F(1,9)=180.63, p<.01]$. Chick continued to make more mean presses on the R-cue, 5.25, than on the Fcue, 1.80 , and this difference was very reliable $[F(1,9)$ $=726.30, p<.01]$. In the case of Dozy, .80 mean presses were made on the $R$-cue and .86 mean responses were made on the F-cue, and these means did not differ significantly $(F<1.0)$.

When monkeys were returned to delayed matching with different items appearing on every trial, the directed forgetting effect found in Experiment $3 b$ disappeared immediately. Whatever processes were at work to produce the effect in Experiment $3 b$ were not carried over to Experiment 3c. It appears that the ability of R- and F-cues to direct differential processing of the memories of sample stimuli may be dependent upon the use of a small set of sample stimuli.

\section{GENERAL DISCUSSION}

The experiments reported here were performed in an attempt to demonstrate the directed forgetting phenomenon in monkeys. They were based on observations made with pigeons, in which repeatedly presenting an F-cue and omitting the comparison stimuli after a sample stimulus led to poor retention of the sample stimulus on F-cued probe trials. The first experiment used a rather complex design in which one or two sample stimuli were presented on a trial, and members of the two-item list could both be R-cued or F-cued or one member could be R-cued and the other F-cued. A simpler design was used in Experiment 2, 
with only one sample stimulus appearing on each trial and R-cues following the sample on half the trials and F-cues following the sample on the other half. An important feature of both Experiment 1 and Experiment 2 was the selection of sample and nonmatching comparison stimuli from a large pool of slides, so that different pictures appeared on each trial within a session. Both experiments were in complete agreement in failing to provide any suggestion of the directed forgetting effect. Monkeys performed equally well on F-cued probe trials and R-cued trials, even when the retention interval was made as long as $16 \mathrm{sec}$.

The disparity between the findings of these first two experiments with monkeys and those reported with pigeons could be explained in two ways. One possibility is that of a species difference in information processing; for example, pigeons might learn to process sample stimuli followed by $R$ - and F-cues differently, while monkeys do not. The other possibility is that the use of a restricted set of sample stimuli, typical of pigeon studies, is necessary to produce the effect. Since different stimuli were used on every trial within a session in the first two monkey experiments, this hypothesis would anticipate a failure to find the directed forgetting effect under these circumstances. The hypothesis also suggests that the directed forgetting effect should appear in monkeys if only a small set of sample stimuli is used. This prediction was tested in Experiments $3 a$ and $3 b$ by looking for the directed forgetting effect with only two stimuli presented alternately as sample and nonmatching comparison stimuli. When colored geometric patterns were used as stimuli in Experiment 3a, performance generally was low and directed forgetting was not found. Two complex pictures from the slide pool were used in Experiment 3b, and clear evidence of directed forgetting appeared. When monkeys were retested with different slides on each trial in Experiment 3c, the subjects immediately performed at equivalent and highly accurate levels on R-cued trials and F-cued probe trials. These findings strongly suggest that the directed forgetting effect may be found only with small sets of sample stimuli and that there is not a species difference between pigeons and monkeys in the way they learn to process R- and F-cued stimuli.

The question then arises as to why demonstration of the directed forgetting effect is dependent upon the use of a small set of sample stimuli. We suggest that this observation is the result of different memory processes used in situations with many and few to-beremembered items. The following notions outline the distinction we wish to make. It is assumed that all sample stimuli presented on delayed matching trials are stored immediately and automatically as episodes in long-term memory (LTM). When test stimuli are presented, they will serve to retrieve memory of the sample stimulus just presented on the current trial. If only two stimuli are used repeatedly over many trials within a session, the comparison stimuli on Trial $n$ will retrieve memories not only of the Trial $n$ sample stimulus but also of the sample and comparison stimuli presented on Trial $n-1, n-2$, etc. To the extent that the animal cannot temporally discriminate the most recent presentation from the earlier ones, errors in choice will be made. That is, PI will act against efficient retention and performance (D'Amato, 1973; Grant, 1975; Roberts, 1980). When different sample stimuli are used on each trial, the test stimuli on Trial $n$ generally will retrieve only memory of the sample stimulus presented on Trial $n$. Therefore, PI will be negligible and accuracy will be high.

In the two-stimulus case, animals will not rely just on retrieval from LTM but will develop an additional within-trial mediating process. This mediating process takes the form of rehearsal of a representation during the retention interval, and the representation indicates which test stimulus should be chosen at the end of the retention interval. This process may be prospective or instructional in nature; that is, the animal may encode the sample into an instruction to respond to a particular test stimulus (Grant, 1981b; Honig \& Thompson, 1982; Roitblat, 1980). Alternatively, an animal might use a more retrospective process of rehearsing a representation of the sample stimulus and then decode this representation into a response instruction at the time the test stimuli are presented (Roberts, 1982). The important point is that an animal develops a small set of stereotyped codes that are rehearsed during the retention interval and that will reliably indicate the correct test stimulus. There are at least two reasons why such codes would be learned. First, the constant repetition of trials with the same stimuli provides ample opportunity for such a coding and rehearsal process to be learned. Second, the maintenance of a reliable guide to correct choice in an active state will override interfering memories retrieved from LTM by the comparison stimuli and thereby provide some insulation against PI.

In order to relate the directed forgetting effect to the use of a rehearsal process, it is held that $R$ - and F-cues develop strong control over the use of mediating codes (Grant, 1981b). On R-cued trials, the code is maintained throughout the delay, whereas on Fcued trials, the mediating process is canceled. When comparison stimuli then appear on an F-cued probe trial, no code is available to direct choice between the comparison stimuli. Pigeon experiments in which the directed forgetting effect has been demonstrated have involved the use of only two sample stimuli (Grant, 1981a; Maki \& Hegvik, 1980; Maki et al., 1981; Stonebraker et al., 1981). This procedure then should 
have allowed for the development of stereotyped codes and for the controlled rehearsal or nonrehearsal of those codes by R-and F-cues.

In the case of Experiments 1 and 2 reported here, we theorize that no coding and rehearsal process developed and that monkeys relied only on the retrieval of information from LTM. That is, comparison stimuli acted to retrieve visual images or salient visual characteristics of the sample just recently stored on a given trial. Since different pictures appeared on every trial, conditions that yield PI were absent, and the need for active maintenance of a code during the retention interval did not arise. Furthermore, we suggest that once visual information about the sample stimulus was stored in LTM, it could not be canceled or modified by F-cues. Given these assumptions, it is then not surprising that F-cues failed to produce loss of retention for pictures in monkeys when the pictures presented were changing constantly from one trial to the next.

The findings of Experiment 3 seem to be in line with these theoretical notions. The sudden introduction of a procedure that used only two sample stimuli should have yielded considerable PI and the accompanying loss of accurate performance. With sufficient practice in Experiment $3 \mathrm{~b}$, the monkeys could have developed appropriate mediating codes, and Rand F-cues could have gained control over the rehearsal of those codes. Indeed, the delayed appearance of the directed forgetting effect in two of the monkeys during probe testing in Experiment 3b may have resulted from the gradual development of control over a mediating rehearsal process by $R$ - and Fcues during the period of testing. The reinstatement of testing with multiple sample stimuli in Experiment 3c should have eliminated both the use of rehearsed codes and the conditions that produce PI. The animals should have returned immediately to the use of visual information retrieved from LTM and thus have shown the sudden rise in accuracy and disappearance of the directed forgetting effect.

In summary, it is suggested that directed forgetting in animals may arise only when the use of a small set of sample stimuli promotes the rehearsal of a small number of stereotyped codes to bridge the retention interval. When a wide variety of stimuli are used, animals will not develop such mediating mechanisms, performance will be based only on the retrieval of visual information about the sample stimuli, and Fcues will have little effect on retention. These findings indicate that training condition, rather than species, is the most important factor determining the appearance of the directed forgetting effect. It may be possible to train pigeons to perform delayed matching with a large variety of sample stimuli (Wright, Santiago, Sands, \& Urcuioli, 1984). We would expect that a directed forgetting experiment performed with pigeons under these conditions would not show differential retention of $\mathbf{R}$ - and Fcued items.

One point that critics of this theoretical position have raised is that it does not seem to articulate well with studies of directed forgetting in humans. Directed forgetting has been found when R-cued and F-cued items were different words appearing on extended lists. Surely, retention in these experiments involves retrieval from LTM and does not involve the short-term rehearsal of fixed codes. This is undoubtedly true, and the directed forgetting effect found with humans must have something to do with differential processing of $\mathbf{R}$ - and F-cued items. Theoretical accounts of directed forgetting in people have emphasized processes that promote differential retrieval of $\mathbf{R}$ - and F-cued items. It has been suggested that $\mathbf{R}$-cued items are better retrieved than F-cued items because (1) $R$-cued items are given longer and more elaborative rehearsal than F-cued items, (2) $R$ cued items are cognitively segregated from F-cued items, and (3) F-cues initiate processes which actively inhibit retrieval of F-cued items (Bjork, 1970; Geiselman, Bjork, \& Fishman, 1983). An important point here is that these processes appear to attenuate retrieval of F-cued items in recall experiments. When recognition memory is used, the difference in retention of R- and F-cued items is either small or nonexistent (Block, 1971; Geiselman, 1974; Geiselman et al., 1983). A further point to be noted is that directed forgetting effects are observed with verbal items. To our knowledge, directed forgetting in humans has not been examined with pictorial recognition memory. Given the weak effects of F-cues in recognition memory for verbal items and the fact that humans have shown excellent long-term recognition memory for very large sets of briefly viewed pictures (Standing, 1973; Standing, Conezio, \& Haber, 1970), it seems very likely that people would not show the directed forgetting effect when recognition memory for pictorial stimuli is involved. If this is the case, then there would be no basic difference between our findings with monkeys in Experiments 1 and 2 and findings with humans in similar experiments. It may be, then, that directed forgetting is not found in humans with all types of memory tests but only under certain circumstances-that is, when retrieval is made difficult by the use of a recall test for verbal items. Similarly, the directed forgetting effect may be found in animals only under certain conditions-that is, when only a small set of to-be-remembered stimuli are used. Furthermore, the processes that yield directed forgetting effects under these different circumstances in animals and humans are quite different.

\section{REFERENCES}

Bjonk, R. A. (1970). Positive forgetting: The noninterference of items intentionally forgotten. Journal of Verbal Learning and Verbal Behavior, 9, 255-268. 
Bjork, R. A., LaBerge, D., \& LeGrande, R. (1968). The modification of short-term memory through instructions to forget. Psychonomic Science, 10, 55-65.

BLock, R. A. (1971). Effects of instructions to forget in shortterm memory. Journal of Experimental Psychology, 89, 1-9.

D'Amato, M. R. (1973). Delayed matching and short-term memory in monkeys. In G. H. Bower (Ed.), The psychology of learning and motivation: Advances in research and theory (Vol. 7). New York: Academic Press.

Geiselman, R. E. (1974). Positive forgetting of sentence material. Memory \& Cognition, 2, 677-682.

Geiselman, R. E., Bjork, R. A., \& Fishman, D. L. (1983). Disrupted retrieval in directed forgetting: A link with posthypnotic amnesia. Journal of Experimental Psychology: General, 112, 58-72.

Grant, D. S. (1975). Proactive interference in pigeon short-term memory. Journal of Experimental Psychology: Animal Behavior Processes, 1, 207-220.

Grant, D. S. (1981a). Stimulus control of information processing in pigeon short-term memory. Learning and Motivation, 12, $19-39$.

Grant, D. S. (1981b). Short-term memory in the pigeon. In N. E. Spear \& R. R. Miller (Eds.), Information processing in animals: Memory mechanisms. Hillsdale, N.J: Erlbaum.

Grant, D. S. (1982). Stimulus control of information processing in rat short-term memory. Journal of Experimental Psychology: Animal Behavior Processes, 8, 154-164.

Grant, D. S. (in press). "Directed forgetting" and proactive interference in pigeons. Canadian Journal of Psychology.

Honig, W. K., \& Thompson, R. K. R. (1982). Retrospective and prospective processing in animal working memory. In G. $\mathrm{H}$. Bower (Ed)., The psychology of learning and motivation (Vol. 16). New York : Academic Press.

Kendrick, D. F., Rilling, M., \& Stonebraker, T. B. (1981). Stimulus control of delayed matching in pigeons: Directed forgetting. Journal of the Experimental Analysis of Behavior, 36, 241-251.

MAK1, W. S. (1981). Directed forgetting in animals. In N. E. Spear \& R. R. Miller (Eds)., Information processing in animals: Memory mechanisms. Hillsdale, N.J: Erlbaum.

Maki, W. S., \& Hegvik, D. K. (1980). Directed forgetting in pigeons. Animal Learning \& Behavior, 8, 567-574.

Maki, W. S., Olson, D., \& REgo, S. (1981). Directed forgetting in pigeons: Analysis of cue functions. Animal Learning \& Behavior, 9, 189-195.

Overman, W. H., \& Doty, R. W. (1980). Prolonged visual memory in macaques and man. Neuroscience, $5,1825-1831$.
Roberts, W. A., (1980). Distribution of trials and intertrial retention in delayed matching to sample with pigeons. Journal of Experimental Psychology: Animal Behavior Processes, 6, 217-237.

Robents, W. A. (1982). Premature closure of controversial issues concerning animal memory representations. The Behavioral and Brain Sciences, 5, 384-385.

Roberts, W. A., \& Kraemer, P. J. (1981). Recognition memory for lists of visual stimuli in monkeys and humans. Animal Learning \& Behavior, 9, 587-594.

RoItblat, H. L. (1980). Codes and coding processes in pigeon short-term memory. Animal Learning \& Behavior, 8, 341-351.

Sands, S. F., \& Wright, A. A. (1980a). Primate memory: Retention of serial list items by a rhesus monkey. Science, 209, 938-939.

Sands, S. F., \& Wright, A. A. (1980b). Serial probe recognition performance by a rhesus monkey and a human with 10 - and 20-item lists. Journal of Experimental Psychology: Animal Behavior Processes, 6, 386-396.

Sands, S. F., \& Wright, A. A. (1982). Monkey and human pictorial memory scanning. Science, 216, 1333-1334.

Standing, L. (1973). Learning 10,000 pictures. Quarterly Journal of Experimental Psychology, 25, 207-222.

Standing, L., Conezio, J., \& Haber, R. N. (1970). Perception and memory for pictures: Single trial learning of 2,560 visual stimuli. Psychonomic Science, 19, 73-74.

Stonebraker, T. B., \& Rilling, M. (1981). Control of delayed matching-to-sample performance using directed forgetting techniques. Animal Learning \& Behavior, 9, 196-201.

Stonebraker, T. B., Rilling, M., \& Kendrick, D. F. (1981). Time dependent effects of double cuing in directed forgetting. Animal Learning \& Behavior, 9, 385-394.

Woodward, A. E., \& BJork, R. A. (1971). Forgetting and remembering in free recall: Intentional and unintentional. Journal of Experimental Psychology, 89, 109-116.

Wright, A. A., Santiago, H. C., Sands, S. F., \& Urcuioli, P. J. (1984). Monkey and pigeon serial probe recognition performance: Effects of item pool size on proactive interference and item specific associations. In $\mathbf{H}$. L. Roitblat, T. G. Bever, \& H. S. Terrace (Eds.), Animal cognition. Hillsdale, NJ: Erlbaum.

(Manuscript recieved November 19, 1982; revision accepted for publication September $9,1983$. 